\title{
Dzyaloshinskii-Moriya driven helical-butterfly structure in $\mathrm{Ba}_{3} \mathrm{NbFe}_{3} \mathrm{Si}_{2} \mathrm{O}_{14}$
}

\author{
V. Scagnoli* \\ Swiss Light Source, Paul Scherrer Institut, CH 5232 Villigen PSI, Switzerland and ETH Zürich, Institut für Quantenelektronik, \\ W. Pauli Strasse 16, 8093 Zürich, Switzerland
}

S. W. Huang, M. Garganourakis, R. A. de Souza, and U. Staub

Swiss Light Source, Paul Scherrer Institut, CH 5232 Villigen PSI, Switzerland

V. Simonet, P. Lejay, and R. Ballou

Institut Néel, CNRS and Université Joseph Fourier, BP166, 38042 Grenoble Cedex 9, France

(Received 23 March 2013; revised manuscript received 17 June 2013; published 18 September 2013)

\begin{abstract}
We have used soft $\mathrm{x}$-ray magnetic diffraction at the $\mathrm{Fe}^{3+} L_{2,3}$ edges to examine to what extent the Dzyaloshinsky-Moriya interaction in $\mathrm{Ba}_{3} \mathrm{NbFe}_{3} \mathrm{Si}_{2} \mathrm{O}_{14}$ influences its low-temperature magnetic structure. A modulated component of the moments along the $c$ axis is present, adding to the previously proposed helical magnetic configuration of coplanar moments in the $a, b$ plane. This leads to a "helical-butterfly" structure and suggests that both the multiaxial in-plane and the uniform out-of-plane Dzyaloshinsky-Moriya vectors are relevant. A nonzero orbital magnetic signal is also observed at the oxygen $K$ edge, which reflects the surprisingly strong hybridization between iron $3 d$ and oxygen $2 p$ states, given the nominal spherical symmetry of the $\mathrm{Fe}^{3+}$ half-filled shell.
\end{abstract}

DOI: 10.1103/PhysRevB.88.104417

PACS number(s): 78.70.Ck, 71.30.+h

\section{INTRODUCTION}

The term chirality was first utilized in science by Lord Kelvin. His original definition has evolved with time and we now speak about a chiral system if such a system exists in two distinct (enantiomeric) states that are interconverted by space inversion, but not by time reversal combined with any proper spatial rotation. ${ }^{1}$ Chirality permeates natural sciences from biochemistry to solid state physics. The fact that living organisms use only the left enantiomers of amino acids is still not well understood. Chirality is also found in magnets. ${ }^{2,3}$ An example is the left or right handedness associated with the helical order of magnetic moments. In principle, the two states are degenerate, resulting in an equipopulation of chiral domains. However, competing interactions or external effects such as strain, can unbalance this ratio, favoring one particular state. In particular, in non-centro-symmetric crystals, characterized by the absence of parity symmetry, a single domain might be selected. Despite having 65 non-centro-symmetric (including 22 chiral) space groups allowing chiral crystal structures, out of 230 , only few single-handed magnetic compounds were reported. ${ }^{4-7}$ Interest in such systems is twofold. First, they can exhibit interesting physical properties such as magnetic Skyrmion lattices ${ }^{8}$ or helimagnons. ${ }^{7}$ The second is related to the discovery of magnetically induced multiferroics ${ }^{9}$ where researchers struggle to find materials with a stronger electrical polarization. ${ }^{10}$ The latter is directly affected by the imbalance between chiral domains, which possess opposite electric polarizations. Therefore materials showing a single chiral domain are promising candidates to host a significant macroscopic electrical polarization, which makes them an ideal model system to study. $\mathrm{Ba}_{3} \mathrm{NbFe}_{3} \mathrm{Si}_{2} \mathrm{O}_{14}$ gathered attention in this respect, exhibiting fully chiral magnetism ${ }^{5}$ and magnetoelectric coupling phenomena. ${ }^{11-13}$

$\mathrm{Ba}_{3} \mathrm{NbFe}_{3} \mathrm{Si}_{2} \mathrm{O}_{14}$ crystallizes in a trigonal $\mathrm{P} 321$ space group $\left(a=b=8.539 \AA, c=5.241 \AA, \gamma=120^{\circ}\right)$. It displays an antiferromagnetic order below $T_{N}=27 \mathrm{~K}$. The magnetic moments are localized on the $\mathrm{Fe}^{3+}$ ions $(L \simeq 0, S=5 / 2)$. These occupy the Wyckoff position (3f) $(0.2496,0,0.5)$ with .2 . site symmetry, forming triangular units in the $a, b$ planes. Elastic neutron scattering studies ${ }^{5}$ suggest that the same triangular configuration of coplanar moments at $120^{\circ}$ from each other is stabilized within each triangle of an $a, b$ plane and that this arrangement is helically modulated from $a, b$ plane to $a, b$ plane along the $c$ axis according to the propagation vector $(0,0, \tau)$ with $\tau$ close to $1 / 7$ [see Fig. 1(a)]. An extremely appealing discovery was that the single crystals are grown enantiopure and that the low-temperature magnetic structure is single domain, with a single chirality of the triangular magnetic arrangement on the triangles and a single chirality of the helical modulation of the magnetic moments, which was dubbed helicity. ${ }^{5}$ It was suggested that the Dzyaloshinsky-Moriya ${ }^{14,15}$ exchange interaction might be responsible for selecting the ground-state configuration $^{5}$ and for the opening of a small gap in the magnetic excitation spectrum. ${ }^{16}$ Another inelastic neutron scattering study proposed the latter might arise as well from single ion anisotropy. ${ }^{17}$ Recent spin resonance experiments support the presence of the Dzyaloshinsky-Moriya interaction, indicating furthermore that not only the uniform component along the $c$ axis of the Dzyaloshinsky-Moriya vector but also its multiaxial component within the $a, b$ plane might be sizable. ${ }^{18}$ The latter could generate an additional component to the magnetic structure not necessarily detected by neutron scattering. To find evidence for such a magnetic motif we have used resonant $\mathrm{x}$-ray diffraction at the $\mathrm{Fe} L$ edges. Our results show clear deviations from the magnetic structure previously proposed, suggesting the existence of such a component.

\section{EXPERIMENTAL DETAILS}

Powders of $\mathrm{Ba}_{3} \mathrm{NbFe}_{3} \mathrm{Si}_{2} \mathrm{O}_{14}$ were synthesized by solid state reaction from stoichiometric amounts of $\mathrm{Nb}_{2} \mathrm{O}_{3}, \mathrm{Fe}_{2} \mathrm{O}_{3}$, 


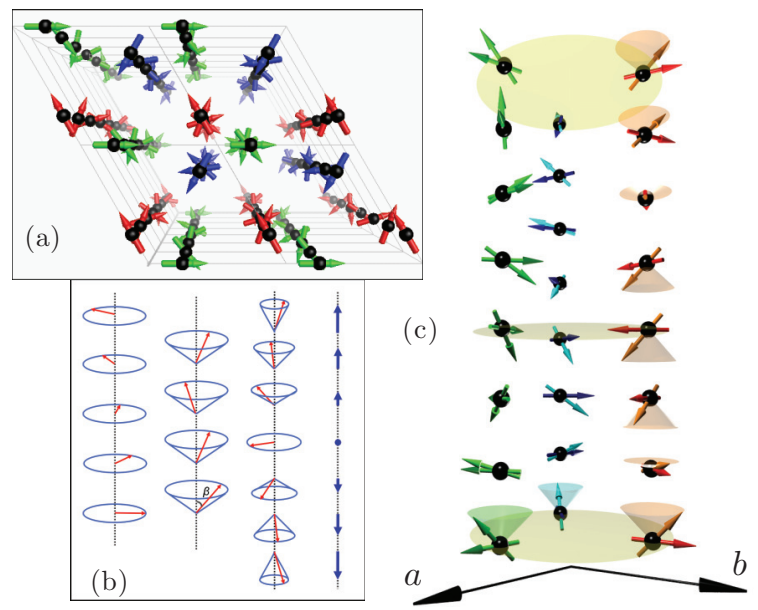

FIG. 1. (Color online) (a) Perspective view along the $c$ axis of the magnetic structure as suggested by neutron diffraction experiments. $\mathrm{Fe}$ ions are in black and different colors are used for the moments on the three Bravais lattices. (b) (From left to right) shows different types of magnetic ordering: a simple spiral, a ferromagnetic (conical) spiral, a complex spiral (or butterfly), and a static longitudinal wave. (c) Pictorial view of the magnetic structure suggested by the present study. The dark colored moments describe the same pattern as in (a). The light colored moments represent the magnetic structure as the sinusoidal modulation along the $c$ axis is superimposed to the basal helical order previously reported. Cones visualize the rotation of the magnetic moments about the $c$ axis and visualize the change in the modulation amplitude. Shaded areas are parallel to $(00 \ell)$ planes. Note that the tilting out of the $a, b$ plane is exaggerated for clarity.

$\mathrm{SiO}_{2}$ oxides and $\mathrm{BaCO}_{3}$ barium carbonate, at $1150{ }^{\circ} \mathrm{C}$ in air within an alumina crucible. The reagents were carefully mixed and pressed at $1 \mathrm{GPa}$ to form compact cylinders before annealing. The phase purity was checked by x-ray powder diffraction. Single crystals were grown from the as-prepared polycrystalline cylinders by the floating-zone method in an image furnace. ${ }^{19}$ The single crystal used in the present investigation was extracted from the same batch as the one used in Ref. 5 and has the same structural chirality $\epsilon_{T}$, to be precise $\epsilon_{T}=-1$. After polishing the surface perpendicular to the [001] direction it was annealed to improve the surface quality.

We have performed resonant $\mathrm{x}$-ray diffraction experiments at the Fe $L_{2,3}$ edge. These energies correspond to a wavelength of approximately $17 \AA$ and are associated to an electric dipole resonance from the iron $2 p$ to $3 d$ levels. Experiments were performed with the RESOXS chamber $^{20}$ at the X11MA beamline $^{21}$ of the Swiss Light Source. The twin Apple undulators provide linear, horizontal $\pi$ and vertical $\sigma$, and circularly, right $R$ and left $L$, polarized $\mathrm{x}$ rays with a polarization rate close to $100 \%$. The polarization of the diffracted beam was not analyzed. The sample was attached to the cold finger of an He flow cryostat with a base temperature of $7 \mathrm{~K}$. Azimuthal scans were achieved by rotation of the single crystal, with an accuracy of approximately $\pm 5^{\circ}$.

\section{RESONANT X-RAY SCATTERING}

The x-ray cross section for magnetic scattering is normally very small, though at synchrotron photon sources such weak signals are routinely measurable. ${ }^{22-25}$ However, when working close to an atomic absorption edge the magnetic scattering signals are significantly enhanced and are element sensitive. Resonant x-ray diffraction occurs when a photon excites a core electron to empty states, and is subsequently re-emitted when the electron and the core hole recombine. ${ }^{26-28}$ This process introduces anisotropic contributions to the $\mathrm{x}$-ray susceptibility tensor, ${ }^{29-31}$ the amplitude of which increases dramatically as the photon energy is tuned to an atomic absorption edge. In the presence of long-range magnetic order, or a spatially anisotropic electronic distribution, the interference of the anomalous scattering amplitudes may lead to Bragg peaks at positions forbidden by the crystallographic space group. An example of such a resonant enhancement of the diffracted intensity as a function of energy occurring in the vicinity of the Fe $L_{3}$ edge in $\mathrm{Ba}_{3} \mathrm{NbFe}_{3} \mathrm{Si}_{2} \mathrm{O}_{14}$ is given in Fig. 3. $\mathrm{X}$ rays thus prove to be a valid alternative or complementary tool to neutron diffraction for the study of magnetic structures. ${ }^{32-36}$ Its superior resolution in reciprocal space can be advantageous, simplifying for instance the precise determination of incommensurate magnetic phases, which is relevant in cases where the incommensurability is very small. ${ }^{37}$

To understand the content of the x-ray resonant magnetic cross section, it is customary to use the expression first derived by Hannon and Trammell for an electric dipole (E1) event: ${ }^{26-28}$

$F_{\boldsymbol{\epsilon}^{\prime}, \boldsymbol{\epsilon}}^{E 1}=\left(\boldsymbol{\epsilon}^{\prime} \cdot \boldsymbol{\epsilon}\right) F^{(0)}-i\left(\boldsymbol{\epsilon}^{\prime} \times \boldsymbol{\epsilon}\right) \cdot \hat{\mathbf{z}}_{n} F^{(1)}+\left(\hat{\boldsymbol{\epsilon}}^{\prime} \cdot \hat{\mathbf{z}}_{n}\right)\left(\hat{\boldsymbol{\epsilon}} \cdot \hat{\mathbf{z}}_{n}\right) F^{(2)}$,

where the first term contributes to the charge (Thompson) Bragg peak. The second and third terms correspond to magnetic diffraction. $\hat{\mathbf{z}}_{n}$ is a unit vector in the direction of the magnetic moment of the $n$th ion in the unit cell and $\boldsymbol{\epsilon}\left(\boldsymbol{\epsilon}^{\prime}\right)$ describes the polarization state of the incoming (outgoing) $\mathrm{X}$ rays. $F^{(i)}$ depend on atomic properties and determine the strength of the resonance. ${ }^{2,28}$ In an antiferromagnet, the second term produces the first-harmonic magnetic satellites and the third term, which contains two powers of the magnetic moment, produces the second-harmonic magnetic satellites. It shows how the intensity of the magnetic diffraction depends on the motif of the magnetic moments and on the orientation of the sample relative to the incident x-ray polarization state. In particular, a noncollinear magnetic motif is able to produce a different diffraction intensity depending on the helicity of the incident $\mathrm{x}$ rays, e.g., $I_{R} \neq I_{L}$, where $I_{R}$ is the intensity measured with incident right-handed circularly polarized photons and $I_{L}$ for left-handed ones. Rotating the sample about the diffraction wave vector might result in a smooth change of the diffracted intensity which helps to reconstruct the magnetic moment motif. It is worth emphasizing that Eq. (1) is an approximation for the resonant magnetic scattering cross section which, strictly speaking, is only valid for a cylindrical symmetrical environment of the resonant ion. When this approximation does not hold the diffracted intensities must be described as exemplified in Refs. 2,38-42. This subject is partially discussed in Appendix. 


\section{RESULTS}

Once the sample is cooled below the Néel temperature $T_{N}$, superstructure peaks $(0,0, n \tau)$ of order $n$ up to three arise from magnetic ordering and magnetically induced lattice distortions (see Fig. 2). The observation of such reflections is remarkable as, given the magnetic motif suggested by neutron diffraction, they should be absent. They are of resonant nature and they disappear when the energy of the incident $\mathrm{x}$ rays is detuned from the iron $L$ edges (see Fig. 3). Nonresonant magnetic intensity could be zero or too small to be visible. Resonant $\mathrm{x}$-ray diffraction is sensitive to the spin, orbital and charge degrees of freedom. ${ }^{28,43-45}$ In order to assert their origin and refine the magnetic structure, we collected their energy, azimuthal and temperature dependence. Figure 3 shows the energy dependence of the three superstructural peaks collected for $\mathrm{x}$ rays with polarization in the diffraction plane (so-called $\pi$ geometry) and perpendicular to it ( $\sigma$ geometry). They measure the maximum intensity of the diffraction peak at different energies (i.e., energy scans at fixed momentum transfer). The first harmonic peak $(n=1)$ shows equal intensity $\left(I_{\pi}=I_{\sigma}\right)$ for both incident $\mathrm{x}$-rays polarization as the energy of the incident $\mathrm{x}$ rays is swept across the iron $L_{3}$ edge. The ratio $I_{\pi}$ over $I_{\sigma}$ is very close to one and has no significant modulation as the sample is rotated about the diffraction wave vector ${ }^{46}$ $(0,0, \tau)$ (so-called azimuthal-angle rotation), as exemplified in Fig. 4. The second harmonic $(0,0,2 \tau)$ energy dependence has $I_{\pi} \neq I_{\sigma}$. Being associated with small lattice or electron density deformations induced by the magnetic ordering, it is expected to exhibit a $I_{\sigma} / I_{\pi}$ ratio different from one. We do not observe any intensity far from the absorption edge. It indicates that the signal originates from the asymmetry of the electron density that appears below the magnetic ordering temperature, possibly triggered by the antiferromagnetic ordering. We have also collected its azimuthal angle dependence (see Fig. 5). In analogy with the first harmonic peak it shows no modulation, with $I_{\sigma}$ and $I_{\pi}$ constant within the error bars. Such results

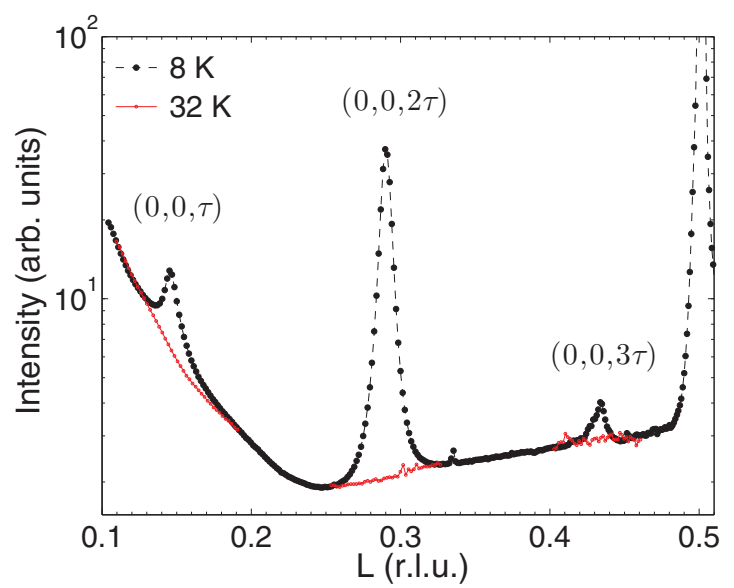

FIG. 2. (Color online) Scan along the [001] direction in reciprocal space at an incident photon energy of $709.8 \mathrm{eV}$ corresponding to the Fe $L_{3}$ edge. r.l.u. denotes reciprocal-lattice units. Dashed (black) line represents data collected at $8 \mathrm{~K}$ while the continuous (red) line represents data collected above $T_{N}$ at $32 \mathrm{~K}$. The peak visible in the vicinity of 0.5 r.l.u. corresponds to higher harmonic contamination from the (001) reflection.

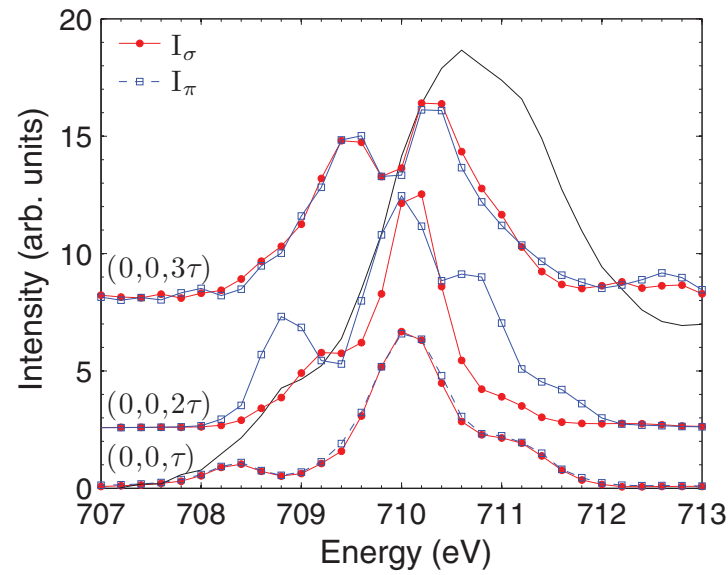

FIG. 3. (Color online) Intensity vs energy of the three satellite reflections in the vicinity of the $\mathrm{Fe} L_{3}$ edge. Spectra collected with incident $\pi$ [(blue) square] and $\sigma$ polarizations [(red) filled circle] at $10 \mathrm{~K}$. Spectra are scaled $[(0,0, \tau)$ and $(0,0,3 \tau)$ were multiplied by 2.5 and 80 , respectively] and shifted for clarity and lines are guides to the eye. The reflectivity contribution has been evaluated and subtracted by performing the same scan above $T_{N}$. The black continuous line represents the sample absorption spectra collected in fluorescent mode.

are supported also by the azimuthal variation of the ratio $I_{\sigma}$ over $I_{\pi}$, which displays smaller error bars due to the elimination of possible systematic errors, which equally affect both intensities, such as misalignments and changes in the sample illuminated area during the azimuthal scan.

Finally, we discuss the third harmonic reflection $(0,0,3 \tau)$. Its energy dependence is quite peculiar. Being $I_{\pi}$ equal to $I_{\sigma}$ suggests the peak to be of magnetic origin, as in the case of $(0,0, \tau)$ reflection. However, the spectral shape differs strongly from the one of the fundamental harmonic. It presents two principal features close in energy rather than

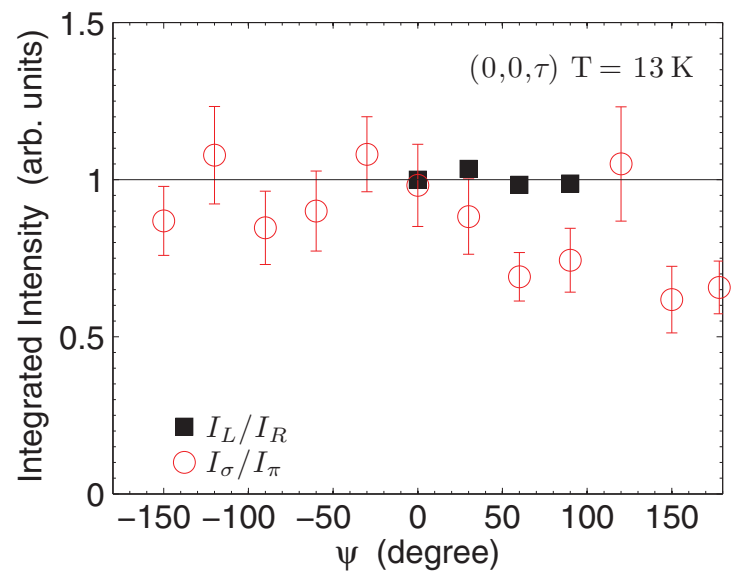

FIG. 4. (Color online) Azimuthal angle dependence of the $I_{\sigma} / I_{\pi}$ (red) and $I_{L} / I_{R}$ (black) ratio for the $(0,0, \tau)$ magnetic reflection. The (black) line represents the predictions of the model described in the text $\left(\chi^{2}=4.0\right.$ for comparison with both data set, $\chi^{2}=1.5$ for the ratio $I_{L} / I_{R}$ alone). Measurements were performed in the vicinity of the $\mathrm{Fe} L_{3}$ edge ( $E=709.8 \mathrm{eV}$ ). The azimuthal angle equals zero when the [100] direction is in the scattering plane. 


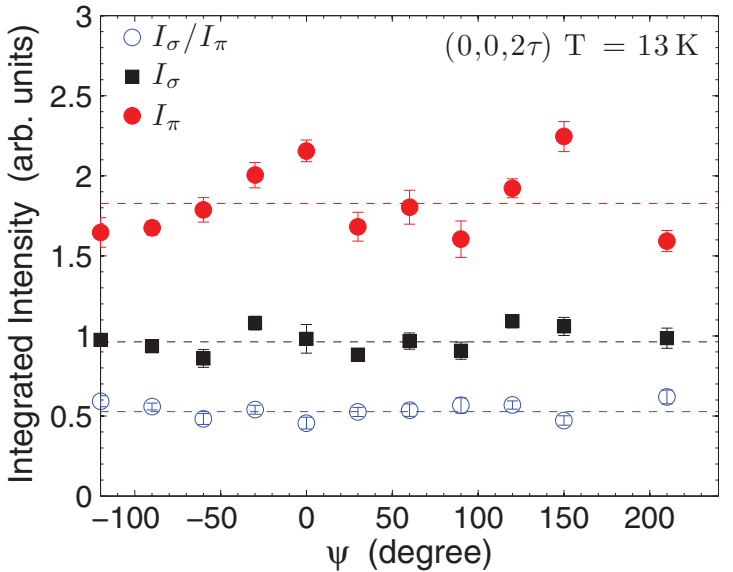

FIG. 5. (Color online) Azimuthal angle dependence of the $(0,0$, $2 \tau)$ superstructural reflection. The line represents a fit to the data with a constant $\left(\chi^{2}=1.6\right.$ for the ratio $\left.I_{\sigma} / I_{\pi}\right)$, as expected form the model presented in the text. Measurements were performed in the vicinity of the $\mathrm{Fe} L_{3}$ edge $(E=709.8 \mathrm{eV})$. The azimuthal angle equals zero when the [100] direction is in the scattering plane.

a single peak with two shoulders as in the case of the $(0$, $0, \tau)$. Absorption effects cannot explain this difference as it would influence more significantly the $(0,0, \tau)$ reflection. At lower angles, the penetration length is reduced as the $\mathrm{x}$ rays have to travel longer into the sample before being diffracted into the detector. As the iron site symmetry (.2.) does not forbid mixed events (e.g., electric dipole-quadrupole), one possible explanation can be a small contribution coming from the electric quadrupole or electric dipole-quadrupole event, ${ }^{28,38,39,47}$ though such contributions are usually expected to be negligible. Such scenario cannot be discarded altogether as the odd reflection intensities are between two and three orders of magnitude smaller compared to other magnetic ordering signal found in oxides..$^{32,34,48-53}$ It was unfortunately not possible to collect the azimuthal angle dependence of the $(0,0,3 \tau)$ reflection due to the weakness of the signal.

The temperature dependence of the satellite reflections (see Fig. 6) shows strong resemblance to the one observed in rare-earth metals. ${ }^{54,55}$ Pursuing the parallel with the rare-earth metals we would expect that the first harmonic arises from magnetic diffraction at the dipole resonance. The second harmonic corresponds to charge or orbital diffraction arising from lattice or electron density modulations. The third-order harmonic might be a magnetic harmonic of the first or might originate from an electric quadrupole resonance, ${ }^{28}$ although such a contribution is expected to be orders of magnitudes weaker. In this case, it could even originate from the presence of higher "multipole" moments (e.g., octupoles) order. This could explain the very different energy dependence between the first and third harmonics, as dipoles and octupoles have different spectral shapes.

Our estimate of the critical exponent $\beta$ found that it is not consistent with mean-field theory. A fit to powerlaw behavior $I_{n \tau} \propto\left(T_{N}-T\right)^{2 \beta_{n}}$ gave an estimate for the critical exponents. They are respectively $\beta_{1}=0.34 \pm 0.04$, $\beta_{2}=0.54 \pm 0.05$, and $\beta_{3}=0.93 \pm 0.08$. In this respect, our system shares similarities with the "basal plane" ordered rare-

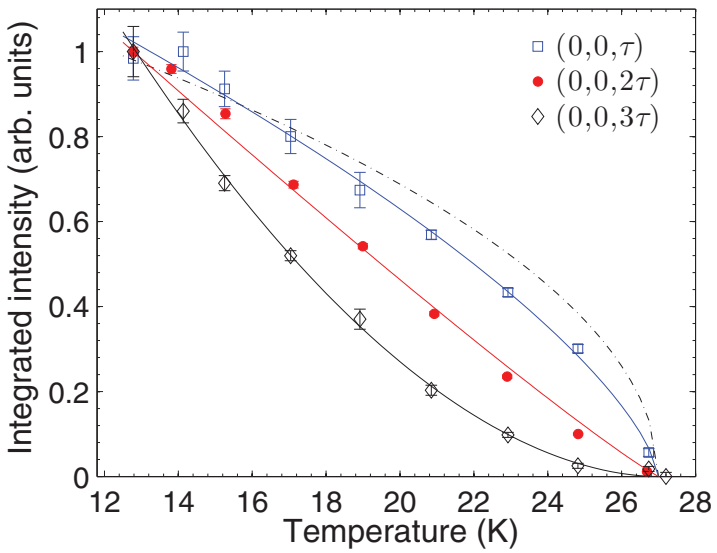

FIG. 6. (Color online) Normalized integrated intensity vs temperature of the three satellite reflections. The solid lines show the best fit to power-law behavior $I_{n \tau} \propto\left(T_{N}-T\right)^{2 \beta_{n}}$. The dashed line is the expected mean-field theory dependence. The $(0,0,2 \tau)$ satellite is seven times more intense than the $(0,0, \tau)$. The same ratio holds between the $(0,0, \tau)$ and the $(0,0,3 \tau)$ satellites. Data were measured with $\pi$ incident photon energy of $710 \mathrm{eV}$.

earth Dy and Ho $\left(\beta_{1}^{\text {Dy }}=0.41 \pm 0.04\right.$ and $\beta_{1}^{\text {Ho }}=0.39 \pm 0.04$, respectively $)^{54,55}$ as opposed to $c$-axis modulated one with $\mathrm{Er}$ and Tm, which follow mean-field theory. It is, however, not possible to push forward the analogy because the origin of the magnetic signal we observe is basically different. It emerges from the deformation by multiaxial Dzyaloshinski-Moriya interactions of a magnetic helix stabilized by twisted exchange interactions, ${ }^{5}$ whereas the rare-earth magnetic orders are rather associated with competing exchange interactions. Another notable difference between the two families of compounds is that in our case the intensity of the second harmonic peak dominates the one of the first harmonic, whilst the opposite is true for the rare earth.

Given the long modulation period of the magnetic structure it was possible to extend our investigation also to the oxygen $K$ edge, which corresponds to an electric dipolar transition from the $1 s$ to the $2 p$ level. Upon cooling below $T_{N}$ a signal is observed at this energy. Figure 7 shows its resonant nature. Observation of a resonant signal on an anion is not unusual. ${ }^{56-58} \mathrm{~A}$ resonant signal can arise, given a nonzero overlap between the initial and the final state, whereas a difference exists in the up/down spin dipolar overlap integrals. The difference can be induced by polarization of the orbitals. ${ }^{59}$ Such an asymmetry can arise also in case of a difference in the lifetime of the up/down spin channels. Recently, Beale et $a l .{ }^{57}$ observed a resonant signal at the oxygen $K$ edge in $\mathrm{TbMn}_{2} \mathrm{O}_{5}$, which they interpreted as a signature of an antiferromagnetically ordered spin polarization on the oxygen site. Such an observation is quite remarkable and we share their opinion that the study of oxygen spin polarization may lead to new insight in the understanding of the magnetoelectric coupling mechanism. As a matter of fact, an antiferromagnetic order at the oxygen site is consistent with neutron diffraction experiments that have already suggested a spin polarization of the oxygen by finding a value of $4 \mu_{B}$ instead of the expected $5 \mu_{B}$ for the spherical $\mathrm{Fe}^{3+}$ half-filled ion magnetic moment. ${ }^{5,11}$ In our case, the signal at the oxygen $K$ edge 


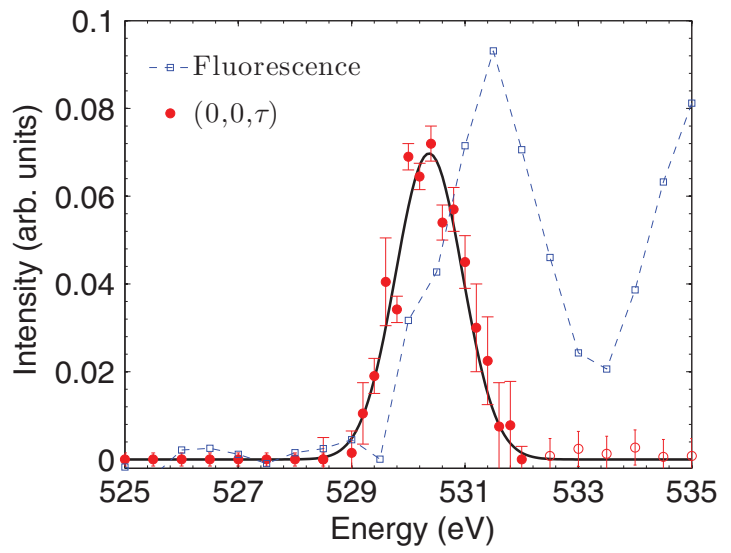

FIG. 7. (Color online) Intensity [(red) circles] vs energy of the $(0,0, \tau)$ satellite reflection at the oxygen $K$ edge collected at $10 \mathrm{~K}$ with $\pi$ incident $x$ rays. The fluorescence spectra [(blue) open squares] obtained in the vicinity is also shown. Full (red) circles: results from a fit of the integrated intensity of a reciprocal lattice scan along the $c^{\star}$ reciprocal lattice direction. Open (red) circles show a result of an energy scan with fix momentum transfer. The (black) continuous line is a Gaussian fit of the oxygen resonance with a FWHM $=1.4 \pm 0.1 \mathrm{eV}$.

is 90 times weaker than the corresponding one observed at the iron $L_{3}$ edge. Note that at the $K$ edge the signal originates solely from the orbital magnetic moment component, given the absence of spin-orbit splitting of the hole in the core state. ${ }^{39,60,61}$ No intensity was observed at the $(0,0,2 \tau)$ and $(0,0,3 \tau)$ satellites at the oxygen $K$ edge.

\section{SYMMETRY CONSIDERATIONS}

Insights into the results can be obtained from group representation analysis, ${ }^{62}$ provided that a single irreducible representation is selected at the magnetic ordering. The analysis is simplified by the fact that the space group P321 associated with the paramagnetic phase is symmorphic. It is, to be precise, a semidirect product of the Abelian translation group associated with a hexagonal lattice and the dihedral point group 32 , which consists of the identity 1 , the anticlockwise rotation $3^{+}$and the clockwise rotation $3^{-}=\left(3^{+}\right)^{2}$ about the ternary $c$ axis and the dyads ( $\pi$ rotations) about the three binary axes at $120^{\circ}$ to each other within the $a, b$ plane. A vector along the reciprocal $c^{\star}$ axis is reversed under the dyads and is invariant otherwise. It follows that the star of the magnetic propagation vector consists of the two vectors $\vec{\tau}_{ \pm}=(0,0, \pm \tau)$ each being associated with the little space group P3, which is a semidirect product of the translation group of the paramagnetic phase and the Abelian cyclic point group 3. An Abelian group $G$ of $n_{G}$ elements has $n_{G}$ conjugacy classes (each being reduced to a singleton owing to the commutativity), which implies that it has $n_{G}$ irreducible representations $\Gamma_{i}\left(i=1, \ldots, n_{G}\right)$. It follows that these are necessarily all of dimension $d_{i}=1$, to comply with the identity $\sum_{i=1}^{n_{G}} d_{i}^{2}=n_{G}$. Each $\Gamma_{i}$ coincides then with its character $\chi_{i}$. The value of $\chi_{i}$ on any group element $g$ is an $n_{G}$ th root $\mathrm{e}^{i 2 \pi p / n_{G}}\left(p=1, \ldots, n_{G}\right)$ of 1 , because the order of $g$ always divide $n_{G}$. The character table is then built by making use of the orthogonality theorems. The basis vector of the invariant subspace of each $\Gamma_{i}$ is also easily deduced by applying the projection operator $\mathcal{P}_{i}=\frac{d_{i}}{n_{G}} \sum_{g \in G} \chi_{i}(g)^{\star} g$ on trial vectors. Table I summarizes such results for the cyclic group 3. The choice of a propagation vector amounts to select an irreducible representation of the translation group and determines a dephasing of moments within each Bravais lattice. Information on the phase relations between moments of distinct Bravais lattices can be extracted only from the irreducible representations of the little co-group. Three Bravais lattices $\mathcal{L}_{v}(v=1,2,3)$ are associated with the positions $(0.2496,0,0.5),(0,0.2496,0.5)$, and $(-0.2496,-0.2496,0.5)$ of the $\mathrm{Fe}^{3+}$ ions on the $3 \mathrm{f}$ site. Under the symmetry operation $3^{+}$, a moment of $\mathcal{L}_{1}$ (respectively, $\mathcal{L}_{2}, \mathcal{L}_{3}$ ) is rotated by an angle of $120^{\circ}$ about the $c$ axis and is transported into $\mathcal{L}_{2}$ (respectively, $\mathcal{L}_{3}, \mathcal{L}_{1}$ ) whereas under the symmetry $3^{-}$it is rotated by an angle of $240^{\circ}$ about the $c$ axis and is transported into $\mathcal{L}_{3}$ (respectively, $\left.\mathcal{L}_{1}, \mathcal{L}_{2}\right)$. This defines a representation $\Gamma$ of the cyclic group 3 of dimension 9 whose character $\chi$ takes the values $\chi(1)=9$, $\chi\left(3^{+}\right)=0$ and $\chi\left(3^{-}\right)=0$ on the group elements. $\Gamma$ reduces into irreducible components as: $\Gamma=3 \Gamma_{1} \oplus 3 \Gamma_{2} \oplus 3 \Gamma_{3}$.

A magnetic structure can be most generally regarded as composed of several sine-wave amplitude modulations of moments: $\frac{1}{2}\left(\vec{v}_{v}\left(\theta_{v}, \phi_{v}\right) e^{-i \xi_{v}} e^{-i \vec{\tau}_{ \pm} \cdot \vec{r}_{v n}}+\right.$ c.c. $)$, where $\vec{r}_{v n}=\vec{r}_{v}+$ $\vec{R}_{n}$ defines the position of the moment of $\mathcal{L}_{v}$ in the $n$th unit cell, $\xi_{v}$ stands for an initial phase, and c.c. means to take the complex conjugate. The reduction of $\Gamma$ then suggests that, whatever the selected irreducible representation $\Gamma_{i}$, three independent directions of the moments are allowed by symmetry and can be combined, for instance along two orthogonal unit vectors in the $a, b$-plane, $\hat{x}_{v}=\left(\pi / 2, \phi_{v}\right)$ at an angle $\phi_{v}$ from the $a$-axis and $\hat{y}_{v}=\left(\pi / 2, \phi_{v}+\pi / 2\right)$ at an angle $\phi_{\nu}+\pi / 2$ from the $a$ axis, and along the unit vector $\hat{z}_{v}=(0,0)$ of the $c$ axis, with possibly vectors $\vec{v}_{v}\left(\theta_{v}, \phi_{v}\right)$ of different lengths.

\section{DISCUSSION}

It was shown, ${ }^{5}$ from collected neutron diffraction intensities, that a helicoidal modulation is stabilized within each $\mathcal{L}_{v}$, associated with a combination of the form $\vec{v}_{v}\left(\pi / 2, \phi_{\nu}\right) e^{-i \xi_{v}}+\sigma \epsilon_{H} \vec{v}_{v}\left(\pi / 2, \phi_{v}+\pi / 2\right) e^{-i\left(\xi_{v}-\pi / 2\right)}=$ $m_{a, b}\left(\hat{x}_{v}+i \sigma \epsilon_{H} \hat{y}_{v}\right) e^{-i \xi_{v}}$ with $\sigma=+1$ for $\vec{\tau}_{+}$and $\sigma=-1$ for $\vec{\tau}_{-}$. It is implicitly assumed that the vectors $\vec{v}_{v}\left(\pi / 2, \phi_{v}\right)=$ $m_{a, b} \hat{x}_{v}$ and $\vec{v}_{v}\left(\pi / 2, \phi_{v}+\pi / 2\right)=m_{a, b} \hat{y}_{v}$ have the same length $m_{a, b}$, which leads to a circular helix. An elliptic helix would have been obtained otherwise, which a priori cannot be excluded. $\epsilon_{H}= \pm 1$ defines the magnetic helicity, that is to say the sense of the rotation of the moments in the helix as one moves along the propagation vector: $\vec{m}\left(\vec{r}_{v n}\right) \times \vec{m}\left(\vec{r}_{v n}+\vec{c}\right)$ $=\epsilon_{H} m_{a, b}^{2} \sin (2 \pi \tau)(\vec{c} /|\vec{c}|)$ whatever the chosen description between $\vec{\tau}_{+}$and $\vec{\tau}_{-}{ }^{63}$ Table I illustrates that a triangular configuration of the moments on each triangle is associated with $\Gamma_{1}$ with a magnetic triangular chirality +1 , that is to say with an anticlockwise sense of the rotation of the moments as one moves anticlockwise on a triangle. A triangular configuration of the moments on each triangle with the opposite magnetic triangular chirality -1 , that is to say with a clockwise sense of the rotation of the moments as one moves anticlockwise on a triangle, emerges from $\Gamma_{2}$ (respectively, $\Gamma_{3}$ ) when $\epsilon_{H}=+1$ (respectively, $\epsilon_{H}=-1$ ), in which case $\Gamma_{3}$ (respectively, $\Gamma_{2}$ ) describes a ferrocollinear configuration of the moments on 
TABLE I. Irreducible representations of the cyclic point group 3, little co-group of the propagation vectors $\vec{\tau}_{ \pm}=(0,0, \pm \tau)$ in the space group P321, and associated invariant basis vectors. $\vec{v}_{v}(\theta, \phi)$ symbolizes a vector associated to a Bravais lattice $\mathcal{L}_{v}$ at an angle $\theta$ from the $c$ axis and the projection of which in the perpendicular plane is at an angle $\phi$ from the $a$ axis. The last column shows the magnetic structure factor for the $(0,0, \pm \tau)$ reflection for each irreducible representation.

\begin{tabular}{cccccc}
\hline \hline & \multicolumn{3}{c}{ Characters } & & \\
\cline { 2 - 4 } & 1 & $3^{+}$ & $3^{-}$ & Basis Vectors & $F_{m}^{\Gamma}(0,0, \pm \tau)$ \\
\hline$\Gamma_{1}$ & 1 & 1 & 1 & $\sum_{v=1}^{3} \vec{v}_{v}\left(\theta, \phi+(v-1) \frac{2 \pi}{3}\right)$ & $\left(0,0, f_{z}\right)$ \\
$\Gamma_{2}$ & 1 & $e^{i \frac{2 \pi}{3}}$ & $e^{i \frac{4 \pi}{3}}$ & $\sum_{v=1}^{3} e^{-i(v-1) \frac{2 \pi}{3}} \vec{v}_{v}\left(\theta, \phi+(v-1) \frac{2 \pi}{3}\right)$ & $\left(f_{x}, f_{y}, 0\right)$ \\
$\Gamma_{3}$ & 1 & $e^{i \frac{4 \pi}{3}}$ & $e^{i \frac{2 \pi}{3}}$ & $\sum_{v=1}^{3} e^{-i(v-1) \frac{4 \pi}{3}} \vec{v}_{v}\left(\theta, \phi+(v-1) \frac{2 \pi}{3}\right)$ & $\left(f_{x}^{\prime}, f_{y}^{\prime}, 0\right)$ \\
\hline \hline
\end{tabular}

each triangle. Intensity asymmetry of the pairs $\vec{K} \pm \vec{\tau}$ of magnetic satellites about reciprocal nodes $\vec{K}$ indicated that for a left-handed structural chirality $\epsilon_{T}=-1$, if $\epsilon_{H}=-1$ then $\Gamma_{1}$ is selected and if $\epsilon_{H}=+1$ then $\Gamma_{2}$ is selected. This interdependence of the dephasing of moments within and between the Bravais lattices $\mathcal{L}_{v}$ was explained as arising from the twist in the exchange paths connecting the moments of consecutive $a, b$ planes, which depends on the structural chirality $\epsilon_{T}$ and imposes the magnetic triangular chirality $\epsilon_{T} \epsilon_{H}$. X-ray anomalous scattering confirmed that the structural chirality of the investigated crystal is $\epsilon_{T}=-1$. Neutron spherical polarimetry finally demonstrated that only the magnetic helicity $\epsilon_{H}=-1$, and therefore only the $\left(\epsilon_{H}, \epsilon_{T} \epsilon_{H}\right)=(-1,+1)$ magnetic helicity-triangular chirality pair, is selected, which was ascribed to the uniform Dzyaloshinsky-Moriya interactions with the Dzyaloshinsky-Moriya vectors all along the $c$ axis. This model ${ }^{5}$ was later confirmed by polarized neutron inelastic scattering with polarization analysis, which allowed probing both the symmetric and antisymmetric nature of the dynamical correlations associated with the magnon excitations emerging from the magnetic order. ${ }^{16}$

A crucial point of the reported model of the circular helices with moments within the $a, b$ plane is that the dephasing of the moments associated with the triangular configuration of moments on each triangle leads to zero magnetic structure factors at the scattering vectors $(0,0, \pm \tau)$. One, however, may recall that the neutrons detect only the components of the moments perpendicular to the scattering vectors. An additional sine-wave amplitude modulated component along the $c$-axis of the moments is therefore not to be excluded. ${ }^{64}$ The length $m_{c}$ of the vector $\vec{v}_{v}(0,0)$ corresponding to the c-axis modulation should, however, be small enough so that the neutron intensities to which it should give rise at the other scattering vectors, $(h, k, \ell \pm \tau)$ with $h \neq 0$ or $k \neq 0$, are drowned beneath the statistical uncertainties of the neutron intensities associated with the main helical modulation component. Table I actually illustrates that this $c$ component of the moments would lead to a zero magnetic structure factor for the scattering vectors $(0,0, \pm \tau)$, and therefore would no longer be detected by resonant $\mathrm{x}$-ray scattering, if the stabilized irreducible representation is either $\Gamma_{2}$ or $\Gamma_{3}$. A nonzero magnetic structure factor vectorially oriented along the $c$ axis is computed only in the case of the irreducible representation $\Gamma_{1}: F_{m}^{\Gamma_{1}}=\left(0,0, f_{z}\right)$. The magnetic intensity $I_{\epsilon^{\prime} \epsilon}=F_{\epsilon^{\prime} \epsilon} F_{\epsilon^{\prime} \epsilon}^{*}$ (* stands for complex conjugation) in the different diffraction channels ${ }^{65}\left(\epsilon=\sigma, \pi\right.$ and $\left.\epsilon^{\prime}=\sigma^{\prime}, \pi^{\prime}\right)$, associated with this amplitude modulated $c$ component, can be calculated with the help of Eq. (1) leading to

$$
\begin{aligned}
& I_{\sigma^{\prime} \sigma}=I_{\pi^{\prime} \pi}=0, \\
& I_{\pi^{\prime} \sigma}=I_{\sigma^{\prime} \pi} \propto \sin ^{2} \theta_{B},
\end{aligned}
$$

where $\theta_{B}$ is the Bragg angle. Noteworthy is the absence of any azimuthal dependence. We therefore expect no modulation of the intensity as we rotate the sample about the scattering wave vector. Moreover, we expect $I_{\sigma}=\left(I_{\sigma^{\prime} \sigma}+\right.$ $\left.I_{\pi^{\prime} \sigma}\right)=I_{\pi}=\left(I_{\sigma^{\prime} \pi}+I_{\pi^{\prime} \pi}\right)$ and $I_{R}=I_{L}$. The latter equality can be derived from Eq. (A1) in Ref. 66, which states $I_{R}-I_{L}=\operatorname{Im}\left\{F_{\sigma^{\prime} \pi}^{*} F_{\sigma^{\prime} \sigma}+F_{\pi^{\prime} \pi}^{*} F_{\pi^{\prime} \sigma}\right\}$.

Another deviation of the magnetic structure might arise from a slight ellipticity of the helices, but according to Table I this would remain invisible in the case of the irreducible representation $\Gamma_{1}$. A finite magnetic structure factor, either $F_{m}^{\Gamma_{2}}=\left(f_{x}, f_{y}, 0\right)$ or $F_{m}^{\Gamma_{3}}=\left(f_{x}^{\prime}, f_{y}^{\prime}, 0\right)$, for the scattering vectors $(0,0, \pm \tau)$ would be obtained only if either the $\Gamma_{2}$ irreducible representation or the $\Gamma_{3}$ irreducible representation were to be stabilized as the main helical modulation component of the magnetic structure, but this is ruled out from the neutron diffraction data.

A mixing of the irreducible representation $\Gamma_{1}$ with the irreducible representation $\Gamma_{2}$ (or $\Gamma_{3}$ ) finally is a priori not to be excluded, though this would imply that the magnetic transition is necessarily first order. Nevertheless, the additional magnetic component should be extremely tiny to escape standard powder neutron detection, since it should lie in the $a, b$ plane to produce a nonzero magnetic structure factor. In the case of the ferrocollinear configuration in the $a, b$ plane, associated with irreducible representation $\Gamma_{2}$ for $\epsilon_{H}=-1$, which gives rise to a magnetic structure factor of the form $F_{m}^{\Gamma_{2}}=\left(f_{x}, f_{y}, 0\right)$, one calculates with the help of Eq. (1) the intensities

$$
\begin{aligned}
& I_{\sigma^{\prime} \sigma}=0, \\
& I_{\pi^{\prime} \sigma}=I_{\sigma^{\prime} \pi}=k_{1} \cos ^{2} \theta_{B}, \\
& I_{\pi^{\prime} \pi}=k_{2} \sin ^{2}\left(2 \theta_{B}\right),
\end{aligned}
$$

where the constants $k_{i}$ depend on the amplitude of the component of the moments associated with the irreducible representation $\Gamma_{2}$ and their orientation in the $a, b$ plane with respect to the moments associated with the main irreducible representation $\Gamma_{1}$. Even in this case, there is no azimuthal angle dependence, but we find $I_{\sigma}<I_{\pi}$ and $I_{R} \neq I_{L}$. Including both $\Gamma_{1}$ and $\Gamma_{3}$ contributions will lead to an azimuthal angle dependence in the rotated channels and again $I_{\sigma}<I_{\pi}$ and $I_{R} \neq I_{L}$.

We are now in the position to compare the $\mathrm{x}$-ray experimental data with the prediction from representation theory. 
Figure 4 shows that the ratio $I_{R}$ over $I_{L}$ is constant as a function of the azimuthal angle and equals one. Also the ratio $I_{\sigma}$ over $I_{\pi}$ is roughly constant within the error bars and is very close to one. It is thus clear that no mixing of irreducible representations is detected and that the magnetic structure abides by only the irreducible representation $\Gamma_{1}$ but involves components of the moments along the three orthogonal direction in space. As a whole, it consists of moments in a triangular arrangement on each triangle in the $a, b$ plane helically modulated along the $c$ axis and exhibiting small up and down oscillations along the $c$ axis in phase with each other and with the same period as the helical modulation, as depicted for a single helix in Fig. 1(b) and for the three lattices in Fig. 1(c). Such a motif is reminiscent of the beatings of butterfly wings (although these wings here are three in number and not four), which lead us to dub it as "helical butterfly." The existence of the butterfly component is consistent with the Dzyaloshinsky-Moriya interactions. Owing to the presence of the three twofold axes at $120^{\circ}$ of each other in the $a, b$ plane, each being perpendicular to one of the three sides of every triangle of moments, the Dzyaloshinsky-Moriya vector associated with each pair of moments must by symmetry lie within the plane containing the link connecting the two moments. ${ }^{67}$ The Dzyaloshinsky-Moriya vector field may therefore have a uniform component along the $c$ axis and a multiaxial component along the side of each triangle. It is this last component that gives rise to the butterfly component. It has been suggested that its contribution might be significant ${ }^{68}$ if not dominating. ${ }^{18}$

Let us now analyze the azimuthal-angle dependence of the $(0,0,2 \tau)$ reflection. According to the $\Gamma_{1}$ magnetic structure factor $F_{m}^{\Gamma_{1}}=\left(0,0, f_{z}\right)$ and the formalism to calculate magnetic diffraction intensity in Ref. 28 we should observe intensity only in the unrotated $\pi^{\prime} \pi$ scattering channel which is at odds with the data shown in Fig. 5. To reconcile the observations with theoretical prediction, we must adopt a more sophisticated model which does not rely on the fact that the resonant ion environment is cylindrically symmetrical. We need a tensorial structure factor $\Psi_{Q}^{K}$, where the positive integer $K$ is the rank of the tensor, and the projection $Q$ can take the $(2 K+1)$ integer values which satisfy $-K \leqslant Q \leqslant K$. For a dipole transition, tensors up to rank 2 contribute $(K \leqslant 2)$. $K=0$ reflects charge contribution, $K=1$ time-odd dipole, and $K=2$ time-even quadrupole. For our superstructural reflection, we are interested in the quadrupolar contribution and given the presence of the threefold axis parallel to the $c$-axis we have $\Psi_{Q}^{K}(0,0,2 \tau)=$ $(-1)^{2 \tau}\left\langle T_{Q}^{K}\right\rangle[1+2 \cos (Q \alpha)]$, which is nonzero only for $Q=$ $0 .\left\langle T_{Q}^{K}\right\rangle$ is an atomic tensor that describes the contribution of each atom to the structure factor. Making use of the formula in Appendix C of Ref. 41, we obtain the following results for the structure factor in the different polarization channels:

$$
\begin{aligned}
F_{\sigma^{\prime} \sigma} & =-\frac{1}{\sqrt{6}} \Psi_{0}^{2}, \\
F_{\pi^{\prime} \sigma} & =F_{\sigma^{\prime} \pi}=0, \\
F_{\pi^{\prime} \pi} & \propto \frac{1}{\sqrt{6}}\left(1+\cos ^{2} \theta_{B}\right) \Psi_{0}^{2}, \\
F_{\sigma} / F_{\pi} & =-1 /\left(1+\cos ^{2} \theta_{B}\right) .
\end{aligned}
$$

A derivation of such relations is presented in Appendix. Such a model suggests no azimuthal dependence in all the diffraction channels and a ratio $I_{\sigma} / I_{\pi}=0.6$ in relative agreement with the azimuthal dependence shown in Fig. 5 with a $\chi^{2}=6.1$. Agreement is improved $\left(\chi^{2}=2.2\right)$ by letting the ratio value vary as a free parameter, with the experimental value of $0.54 \pm 0.02$, still reasonably close to the one derived by Eq. (4). However, such a ratio, as exemplified in Fig. 3, is not constant as a function of energy. These deviations might arise from a small symmetry break resulting in a loss of the threefold axis which would cause extra terms to appear in the structure factor. The latter has also been suggested recently by terahertz spectroscopy. ${ }^{69}$ Experimental uncertainties are however too big to extract more quantitative conclusions on the presence of such contributions.

\section{CONCLUSION}

We have studied the magnetic structure of the intriguing compound $\mathrm{Ba}_{3} \mathrm{NbFe}_{3} \mathrm{Si}_{2} \mathrm{O}_{14}$ with resonant x-ray diffraction at the $\mathrm{Fe} L$ edges and $\mathrm{O} K$ edge. These experiments give new insight into the details of the magnetic structure recently determined by neutron diffraction. Our experiments provide evidence of an extra sinusoidal modulation of the Fe magnetic moments along the crystallographic $c$ axis, concomitant with the helical order in the $a, b$ plane, generating an helicalbutterfly magnetic structure. Such sinusoidal modulation arises from the Dzyaloshinsky-Moriya interaction as suggested by symmetry consideration and recent linear spin-wave theory calculations. ${ }^{68}$ The orbital magnetic signal observed at the oxygen $K$ edge reflects the strong hybridization between iron $3 d$ and oxygen $2 p$ states. Finally, the energy dependence of $I_{\sigma} / I_{\pi}$ ratio for the $(0,0,2 \tau)$ reflection hints to a possible symmetry break with loss of the threefold axis, however, $a b$ initio calculation would be needed to obtain quantitative information.

\section{ACKNOWLEDGMENTS}

We would like to thank S. W. Lovesey and J. P. Hill for stimulating discussion. This work has been supported by the Swiss National Science Foundation, NCCR MaNEP.

\section{APPENDIX: QUADRUPOLAR STRUCTURE FACTOR}

In analogy with Ref. 41, we obtain expression for $\Psi_{Q}^{K}$, written in the coordinate space $(\mathrm{x}, \mathrm{y}, \mathrm{z})$, as a sum of quantities that are even $\left(A_{Q}^{K}\right)$ and odd $\left(B_{Q}^{K}\right)$ functions of the projection $Q$ with $-K \leqslant Q \leqslant K$. We give an expression analogue to Eq. (B5) of Ref. 41 for a generic $(0,0, \ell)$ reflection:

$$
\begin{gathered}
A_{0}^{0}=\Psi_{0}^{0}, \\
A_{0}^{1}=\frac{1}{\sqrt{2}}\left(\Psi_{-1}^{1}-\Psi_{1}^{1}\right), \\
A_{1}^{1}=\frac{1}{2}\left(\Psi_{-1}^{1}+\Psi_{1}^{1}\right), \\
B_{1}^{1}=\frac{1}{\sqrt{2}} \Psi_{0}^{1}, \\
A_{0}^{2}=\frac{\sqrt{6}}{4}\left(\Psi_{-2}^{2}+\Psi_{2}^{2}\right)-\frac{1}{2} \Psi_{0}^{2},
\end{gathered}
$$




$$
\begin{aligned}
& A_{1}^{2}=\frac{1}{2}\left(\Psi_{-2}^{2}-\Psi_{2}^{2}\right), \\
& B_{1}^{2}=\frac{1}{2}\left(\Psi_{-1}^{2}-\Psi_{1}^{2}\right), \\
& A_{2}^{2}=\frac{1}{4}\left(\Psi_{-2}^{2}+\Psi_{2}^{2}\right)+\frac{\sqrt{6}}{4} \Psi_{0}^{2}, \\
& B_{2}^{2}=\frac{1}{2}\left(\Psi_{-1}^{2}+\Psi_{1}^{2}\right) .
\end{aligned}
$$

Limiting ourselves to the quadrupolar contribution $(K=2)$ and taking advantage of the structure factor $\Psi_{Q}^{2}(0,0,2 \tau)=$ $(-1)^{2 \tau}\left\langle T_{Q}^{2}\right\rangle[1+2 \cos (Q \alpha)]$, we have only $\Psi_{0}^{2}$ different from zero.

Expressions in Eq. (A3) therefore simplify leading to, e.g., $B_{Q}^{2}=0$ and $A_{2}^{2} \propto A_{0}^{2}$. Substituting Eq. (A3) in Eqs. (C1)-(C3) of Ref. 41, one obtains the expression quoted in Eq. (4). *valerios@ethz.ch

${ }^{1}$ M. Avalos, R. Babiano, P. Cintas, J. L. Jimnez, J. C. Palacios, and L. D. Barron, Chem. Rev. 98, 2391 (1998).

${ }^{2}$ S. W. Lovesey and S. P. Collins, X-Ray Scattering and Absorption by Magnetic Materials (Clarendon Press, 1996).

${ }^{3}$ V. Simonet, M. Loire, and R. Ballou, Eur. Phys. J.: Spec. Top. 213, 5 (2012).

${ }^{4}$ M. Ishida, Y. Endoh, S. Mitsuda, Y. Ishikawa, and M. Tanaka, J. Phys. Soc. Jpn. 54, 2975 (1985).

${ }^{5}$ K. Marty, V. Simonet, E. Ressouche, R. Ballou, P. Lejay, and P. Bordet, Phys. Rev. Lett. 101, 247201 (2008).

${ }^{6}$ S. V. Grigoriev, D. Chernyshov, V. A. Dyadkin, V. Dmitriev, S. V. Maleyev, E. V. Moskvin, D. Menzel, J. Schoenes, and H. Eckerlebe, Phys. Rev. Lett. 102, 037204 (2009).

${ }^{7}$ M. Janoschek, F. Bernlochner, S. Dunsiger, C. Pfleiderer, P. Böni, B. Roessli, P. Link, and A. Rosch, Phys. Rev. B 81, 214436 (2010). ${ }^{8}$ S. Mühlbauer, B. Binz, F. Jonietz, C. Pfleiderer, A. Rosch, A. Neubauer, R. Georgii, and P. Bni, Science 323, 915 (2009).

${ }^{9}$ T. Kimura, T. Goto, H. Shintani, K. Ishizaka, T. Arima, and Y. Tokura, Nature (London) 426, 55 (2003).

${ }^{10}$ R. D. Johnson, L. C. Chapon, D. D. Khalyavin, P. Manuel, P. G. Radaelli, and C. Martin, Phys. Rev. Lett. 108, 067201 (2012).

${ }^{11}$ K. Marty, P. Bordet, V. Simonet, M. Loire, R. Ballou, C. Darie, J. Kljun, P. Bonville, O. Isnard, P. Lejay, B. Zawilski, and C. Simon, Phys. Rev. B 81, 054416 (2010).

${ }^{12}$ H. D. Zhou, L. L. Lumata, P. L. Kuhns, A. P. Reyes, E. S. Choi, N. S. Dalal, J. Lu, Y. J. Jo, L. Balicas, J. S. Brooks, and C. R. Wiebe, Chem. Mater. 21, 156 (2009).

${ }^{13}$ C. Lee, E. Kan, H. Xiang, and M.-H. Whangbo, Chem. Mater. 22, 5290 (2010).

${ }^{14}$ I. Dzyaloshinsky, J. Phys. Chem. Solids 4, 241 (1958).

${ }^{15}$ T. Moriya, Phys. Rev. Lett. 4, 228 (1960).

${ }^{16}$ M. Loire, V. Simonet, S. Petit, K. Marty, P. Bordet, P. Lejay, J. Ollivier, M. Enderle, P. Steffens, E. Ressouche, A. Zorko, and R. Ballou, Phys. Rev. Lett. 106, 207201 (2011).

${ }^{17}$ C. Stock, L. C. Chapon, A. Schneidewind, Y. Su, P. G. Radaelli, D. F. McMorrow, A. Bombardi, N. Lee, and S.-W. Cheong, Phys. Rev. B 83, 104426 (2011).

${ }^{18}$ A. Zorko, M. Pregelj, A. Potočnik, J. van Tol, A. Ozarowski, V. Simonet, P. Lejay, S. Petit, and R. Ballou, Phys. Rev. Lett. 107, 257203 (2011).

${ }^{19}$ P. Bordet, I. Gelard, K. Marty, A. Ibanez, J. Robert, V. Simonet, B. Canals, R. Ballou, and P. Lejay, J. Phys.: Condens. Matter 18, 5147 (2006).

${ }^{20}$ U. Staub, V. Scagnoli, Y. Bodenthin, M. García-Fernández, R. Wetter, A. M. Mulders, H. Grimmer, and M. Horisberger, J. Synchr. Rad. 15, 469 (2008).
${ }^{21}$ U. Flechsig, F. Nolting, A. F. Rodriguez, J. Krempasky, C. Quitmann, T. Schmidt, S. Spielmann, and D. Zimoch, in Proceedings of the Tenth International Conference on Synchrotron Radiation Instrumentation SRI 2009, No. 1234 (AIP, NY, 2010), pp. 319-322.

${ }^{22}$ V. Fernandez, C. Vettier, F. de Bergevin, C. Giles, and W. Neubeck, Phys. Rev. B 57, 7870 (1998).

${ }^{23}$ T. Brückel, M. Lippert, T. Köhler, J. R. Schneider, W. Prandl, V. Rilling, and M. Schilling, Acta Crystallogr., Sect. A: Found. Crystallogr. 52, 427 (1996).

${ }^{24}$ J. Strempfer, T. Brückel, U. Rütt, J. R. Schneider, K.-D. Liss, and T. Tschentscher, Acta Crystallogr., Sect. A: Found. Crystallogr. 52, 438 (1996).

${ }^{25}$ W. Neubeck, C. Vettier, F. de Bergevin, F. Yakhou, D. Mannix, L. Ranno, and T. Chatterji, J. Phys. Chem. Solids 62, 2173 (2001).

${ }^{26}$ J. P. Hannon, G. T. Trammell, M. Blume, and D. Gibbs, Phys. Rev. Lett. 61, 1245 (1988).

${ }^{27}$ J. P. Hannon, G. T. Trammell, M. Blume, and D. Gibbs, Phys. Rev. Lett. 62, 2644 (1989).

${ }^{28}$ J. P. Hill and D. F. McMorrow, Acta Crystallogr., Sect. A: Found. Crystallogr. 52, 236 (1996)

${ }^{29}$ D. Templeton and L. Templeton, Acta Crystallogr., Sect. A: Found. Crystallogr. 38, 62 (1982).

${ }^{30}$ V. E. Dmitrienko, Acta Crystallogr., Sect. A: Found. Crystallogr. 39, 29 (1983).

${ }^{31}$ D. Templeton and L. Templeton, Acta Crystallogr., Sect. A: Found. Crystallogr. 42, 478 (1986)

${ }^{32}$ V. Scagnoli, U. Staub, A. M. Mulders, M. Janousch, G. I. Meijer, G. Hammerl, J. M. Tonnerre, and N. Stojic, Phys. Rev. B 73, 100409(R) (2006).

${ }^{33}$ S. B. Wilkins, T. R. Forrest, T. A. W. Beale, S. R. Bland, H. C. Walker, D. Mannix, F. Yakhou, D. Prabhakaran, A. T. Boothroyd, J. P. Hill, P. D. Hatton, and D. F. McMorrow, Phys. Rev. Lett. 103, 207602 (2009)

${ }^{34}$ H. Jang, J.-S. Lee, K.-T. Ko, W.-S. Noh, T. Y. Koo, J.-Y. Kim, K.-B. Lee, J.-H. Park, C. L. Zhang, S. B. Kim, and S.-W. Cheong, Phys. Rev. Lett. 106, 047203 (2011).

${ }^{35}$ R. D. Johnson, S. Nair, L. C. Chapon, A. Bombardi, C. Vecchini, D. Prabhakaran, A. T. Boothroyd, and P. G. Radaelli, Phys. Rev. Lett. 107, 137205 (2011).

${ }^{36}$ V. Scagnoli, M. Allieta, H. Walker, M. Scavini, T. Katsufuji, L. Sagarna, O. Zaharko, and C. Mazzoli, Phys. Rev. B 86, 094432 (2012).

${ }^{37}$ S. Agrestini, C. Mazzoli, A. Bombardi, and M. R. Lees, Phys. Rev. B 77, 140403 (2008).

${ }^{38} \mathrm{P}$. Carra and B. T. Thole, Rev. Mod. Phys. 66, 1509 (1994). 
${ }^{39}$ S. W. Lovesey, E. Balcar, K. S. Knight, and J. F. Rodriguez, Phys. Rep. 411, 233 (2005).

${ }^{40}$ N. Stojic, N. Binggeli, and M. Altarelli, Phys. Rev. B 72, 104108 (2005).

${ }^{41}$ V. Scagnoli and S. W. Lovesey, Phys. Rev. B 79, 035111 (2009).

${ }^{42}$ M. W. Haverkort, N. Hollmann, I. P. Krug, and A. Tanaka, Phys. Rev. B 82, 094403 (2010).

${ }^{43}$ Y. Murakami, H. Kawada, H. Kawata, M. Tanaka, T. Arima, Y. Moritomo, and Y. Tokura, Phys. Rev. Lett. 80, 1932 (1998).

${ }^{44}$ U. Staub, G. I. Meijer, F. Fauth, R. Allenspach, J. G. Bednorz, J. Karpinski, S. M. Kazakov, L. Paolasini, and F. d'Acapito, Phys. Rev. Lett. 88, 126402 (2002).

${ }^{45}$ U. Staub, M. Shi, C. Schulze-Briese, B. D. Patterson, F. Fauth, E. Dooryhee, L. Soderholm, J. O. Cross, D. Mannix, and A. Ochiai, Phys. Rev. B 71, 075115 (2005).

${ }^{46}$ Data are collected for a Bragg angle $\theta_{B}=14.1^{\circ}$ where a significant contribution from specular reflectivity is present. Such a contribution is different for $I_{\pi}$ and $I_{\sigma}$ and, combined with the weakness of the signal, complicates the determination of the magnetic Bragg diffraction contribution. In this respect, the data gathered with incident circularly polarized photons $\left(I_{R}\right.$ and $\left.I_{L}\right)$ provide a more reliable data set, as being a complex combination of the linearly polarized light, they present the same background for $I_{R}$ and $I_{L}$. Indeed, the ratio $I_{L}$ over $I_{R}$ is very close to one over the investigated range and sports smaller error bars.

${ }^{47}$ I. Marri and P. Carra, Phys. Rev. B 69, 113101 (2004).

${ }^{48}$ S. B. Wilkins, P. D. Spencer, P. D. Hatton, S. P. Collins, M. D. Roper, D. Prabhakaran, and A. T. Boothroyd, Phys. Rev. Lett. 91, 167205 (2003).

${ }^{49}$ U. Staub, V. Scagnoli, A. M. Mulders, K. Katsumata, Z. Honda, H. Grimmer, M. Horisberger, and J. M. Tonnerre, Phys. Rev. B 71, 214421 (2005).

${ }^{50}$ M. García-Fernández, V. Scagnoli, U. Staub, A. M. Mulders, M. Janousch, Y. Bodenthin, D. Meister, B. D. Patterson, A. Mirone, Y. Tanaka, T. Nakamura, S. Grenier, Y. Huang, and K. Conder, Phys. Rev. B 78, 054424 (2008).

${ }^{51}$ U. Staub, Y. Bodenthin, C. Piamonteze, M. García-Fernández, V. Scagnoli, M. Garganourakis, S. Koohpayeh, D. Fort, and S. W. Lovesey, Phys. Rev. B 80, 140410 (2009).
${ }^{52}$ R. A. de Souza, U. Staub, V. Scagnoli, M. Garganourakis, Y. Bodenthin, and H. Berger, Phys. Rev. B 84, 014409 (2011).

${ }^{53}$ S. W. Lovesey, V. Scagnoli, M. Garganourakis, S. M. Koohpayeh, C. Detlefs, and U. Staub, J. Phys.: Condens. Matter 25, 362202 (2013).

${ }^{54}$ G. Helgesen, J. P. Hill, T. R. Thurston, D. Gibbs, J. Kwo, and M. Hong, Phys. Rev. B 50, 2990 (1994).

${ }^{55}$ G. Helgesen, J. P. Hill, T. R. Thurston, and D. Gibbs, Phys. Rev. B 52, 9446 (1995).

${ }^{56}$ D. Mannix, A. Stunault, N. Bernhoeft, L. Paolasini, G. H. Lander, C. Vettier, F. de Bergevin, D. Kaczorowski, and A. Czopnik, Phys. Rev. Lett. 86, 4128 (2001).

${ }^{57}$ T. A. W. Beale, S. B. Wilkins, R. D. Johnson, S. R. Bland, Y. Joly, T. R. Forrest, D. F. McMorrow, F. Yakhou, D. Prabhakaran, A. T. Boothroyd, and P. D. Hatton, Phys. Rev. Lett. 105, 087203 (2010).

${ }^{58}$ R. A. de Souza, U. Staub, V. Scagnoli, M. Garganourakis, Y. Bodenthin, S.-W. Huang, M. Garcia-Fernandez, S. Ji, S.-H. Lee, S. Park, and S.-W. Cheong, Phys. Rev. B 84, 104416 (2011).

${ }^{59}$ M. van Veenendaal, J. B. Goedkoop, and B. T. Thole, Phys. Rev. Lett. 78, 1162 (1997).

${ }^{60}$ P. Carra, B. T. Thole, M. Altarelli, and X. Wang, Phys. Rev. Lett. 70, 694 (1993).

${ }^{61}$ J. Luo, G. T. Trammell, and J. P. Hannon, Phys. Rev. Lett. 71, 287 (1993).

${ }^{62}$ R. Ballou and B. Ouladdiaf, in Neutron Scattering from Magnetic Materials, edited by T. Chatterji (Elsevier, 2006), Chap. 3.

${ }^{63}$ If we impose $\phi_{\nu=2,3}-\phi_{1}$ according to Table I, then we must have $\xi_{1}=\xi_{2}=\xi_{3}$, which can be set to 0 , together with $\phi_{1}$, without loss of generality.

${ }^{64}$ In which case, we would rather have the combination $\vec{v}_{v}\left(\pi / 2, \phi_{v}\right) e^{-i \xi_{v}}+\sigma \epsilon_{H} \vec{v}_{v}\left(\pi / 2, \phi_{v}+\pi / 2\right) e^{-i\left(\xi_{v}-\pi / 2\right)}+\vec{v}_{v}(0,0) e^{-i \xi_{v}^{\prime}}=$ $m_{a, b}\left(\hat{x}_{v}+i \sigma \epsilon_{H} \hat{y}_{v}\right) e^{-i \xi_{v}}+m_{c} \hat{z}_{v} e^{-i \xi_{\nu}^{\prime}}$.

${ }^{65} \mathrm{We}$ drop the bold style for $\boldsymbol{\epsilon}\left(\boldsymbol{\epsilon}^{\prime}\right)$ to $\epsilon\left(\epsilon^{\prime}\right)$ for the sake of simplicity.

${ }^{66}$ J. Fernández-Rodríguez, S. W. Lovesey, and J. A. Blanco, Phys. Rev. B 77, 094441 (2008).

${ }^{67}$ T. Moriya, Phys. Rev. 120, 91 (1960).

${ }^{68}$ J. Jensen, Phys. Rev. B 84, 104405 (2011).

${ }^{69}$ L. Chaix, S. de Brion, F. Lévy-Bertrand, V. Simonet, R. Ballou, B. Canals, P. Lejay, J. B. Brubach, G. Creff, F. Willaert, P. Roy, and A. Cano, Phys. Rev. Lett. 110, 157208 (2013). 